\title{
Resúmenes de los proyectos del Fondo de Investigación, UCA 2012-2013
}

\section{Proyecto I \\ Desarrollo de un prototipo de aislador sísmico de bajo costo para estructuras de bajo peso.}

\section{Unidad responsable}

- Departamento de Mecánica Estructural.

\section{Equipo de Investigación}

- Erick Burgos Ganuza, Investigador principal, Departamento de Mecánica Estructural.

- Alba Fidelina Alfaro, Co-investigadora, Departamento de Mecánica Estructural.

- Ernesto Pérez Claros, Asistente de investigación, Departamento de Mecánica Estructural.

- Valeria Flores Ramos, Asistente de investigación, Departamento de Mecánica Estructural.

- Miguel Ángel Rubio, Asistente de investigación, Departamento de Mecánica Estructural.

\section{Resumen}

En el presente proyecto se propone diseñar y fabricar un aislador sísmico prototipo de bajo costo, empleando materiales reciclados para ser utilizado en estructuras de bajo peso. Con ello se procura caracterizar mecánicamente tres elementos: el acero de refuerzo determinando su módulo de elasticidad y capacidad de deformación; la mezcla de poliuretano-caucho reciclado determinando su módulo de elasticidad, elongación última, esfuerzo de compresión y módulo de rigidez a corte; y los polímeros investigados en el mercado, a saber, compuesto flexible de policloruro de vinilo (PVC) y hule termoplástico estirénico. También se aspira estudiar el comportamiento dinámico del prototipo de aislador, determinando su capacidad de disipación de energía, su rigidez horizontal y su curva de histéresis. Adicionalmente 
se realiza una investigación en el mercado salvadoreño sobre los polímeros existentes en el país.

\section{Proyecto 2}

\section{Un análisis alternativo sectorial desde la perspectiva insumo-producto para El Salvador y su evolución en el período 1990-2006.}

\section{Unidad responsable}

- Departamento de Economía.

\section{Equipo de Investigación}

- Mario César Sánchez, Investigador, Departamento de economía.

- Marielos García, Asistente de investigación, Departamento de economía.

- Carla Catalina Galdámez, Colaboradora estudiante.

\section{Resumen}

El proyecto tiene como objetivos principales, en primer lugar, realizar un análisis sectorial de requerimientos y de eslabonamientos de variables fundamentales, como las exportaciones e importaciones, el producto y el empleo para 1990-2006 mediante el análisis insumo-producto. En segundo lugar, realizar el análisis antedicho pero para variables no convencionales y heterodoxas, como el monto de salarios, beneficios y capital. Además, se realiza un análisis del impacto de la renta agrícola en la tasa de beneficio agregada y sectorial de El Salvador. Finalmente, se propone realizar un análisis de productividad sectorial.

\section{Proyecto 3}

\section{Cadena socio-productiva de suministros y consumo: un estudio de caso en Guaymango y Jujutla.}

\section{Unidades responsables}

- Departamento de Operaciones y Sistemas.

- Departamento de Economía. 


\section{Equipo de Investigación}

- Gerardo Morales, Departamento de Operaciones y Sistemas.

- Fernando Rivas, Departamento de Operaciones y Sistemas.

- Lilian Vega, Departamento de Economía.

- Silvia Margarita Vidal Lara y Fátima Elizabeth Meléndez Martínez, estudiantes de la carrera de Ingeniería Industrial.

- Alejandra Vanessa Escobar Grande, licenciada en Economía.

- Alejandra María Ayala Castellanos, estudiante de la licenciatura en Comunicación Social.

- $\quad \mathrm{Al}$ inicio, también participaron Javier Zaldívar y José Ricardo Ciudad Real, ambos estudiantes de Ingeniería Industrial.

\section{Resumen}

Esta investigación muestra cómo en circuitos de economía social o solidaria se utilizan las herramientas y metodologías para optimizar los procesos de producción, distribución, circulación y consumo de valores de uso, y cómo el diseño de estos procesos se ejecuta utilizando los métodos de ingeniería de procesos, de economía y la investigación-acción participativa. Se dice participativa en la medida que las personas que forman parte de las comunidades, quienes son objetos de investigación, se convierten a su vez en sujetos, ya que ponen sus saberes a disposición del equipo de investigación, y así de manera conjunta se construye conocimiento y propuestas de acción para resolver problemas de suministros y consumos básicos de la comunidad.

\section{Proyecto 4}

\section{Compilación, análisis y publicación de comentarios breves de coyuntura política, escritos por Ignacio Ellacuría (1969-1979).}

\section{Unidad responsable}

- Departamento de Filosofía.

\section{Equipo de Investigación}

- Luis Alvarenga, responsable, Departamento de Filosofía.

- Carlos Mauricio Hernández, Departamento de Filosofía.

- Roberto Valdés Valle, Investigador, Departamento de Filosofía.

- Miguel Saúl Rivas, Investigador, Departamento de Filosofía.

- Héctor López, Asistente, Departamento de Filosofía. 


\section{Resumen}

El proyecto se enfoca en recopilar, organizar y analizar los escritos breves de coyuntura que Ignacio Ellacuría elaboró entre 1969 y 1979 sobre la realidad política, social y eclesial de El Salvador. La mayoría de estos artículos fueron leídos entre 1978 y 1979, en la radio YSAX del Arzobispado de San Salvador; y otros fueron publicados en la revista Estudios Centroamericanos (ECA) de la Universidad Centroamericana "José Simeón Cañas" (UCA) y en el semanario Orientación, también del Arzobispado de San Salvador.

\section{Proyecto 5}

\section{Estética y política: modernización cultural en El Salvador (1940-1980).}

\section{Unidades responsables}

- Departamento de Comunicaciones y Cultura.

- Departamento de Organización del Espacio y Audiovisuales.

\section{Equipo de Investigación}

- Ricardo Roque Baldovinos, Coordinador e investigador, Departamento de Comunicaciones y Cultura.

- José Manuel González, Investigador, Departamento de Comunicaciones y Cultura.

- Sandra Gutiérrez Poizat, Investigadora, Departamento de Organización del Espacio.

- Nataly Guzmán, Investigadora, Departamento de Comunicaciones y Cultura.

- Fernando Martínez Barrios, Asistente de investigación, Departamento de Comunicaciones y Cultura.

- Rebeca Monge, Asistente de investigación, Departamento de Comunicaciones y Cultura.

- Sofía Rivera, Asistente de investigación, Departamento de Organización del Espacio.

- Julio Sánchez, Asistente de investigación, Departamento de Comunicaciones y Cultura. 


\section{Resumen}

Este proyecto aborda la historia reciente de El Salvador a través de un estudio de la modernización cultural como vía de entrada para entender, al menos en parte, los cambios en la sensibilidad que tienen lugar durante un período comprendido entre las décadas de 1940 y 1980 . Este período se caracteriza por una aceleración del proceso de modernización, que si bien fue conducido por regímenes autoritarios de corte militar, supuso la apertura de nuevos espacios de socialización y de expresión democráticos que fueron la base del proceso revolucionario que desembocó en la guerra de 19791992. El objetivo de este estudio ha sido pues, documentar y comprender esta nueva dinámica cultural donde se destacan el peso creciente de la industria cultural mediática en determinar la experiencia cotidiana, la reconfiguración del espacio de la ciudad con el auge de propuestas modernistas de urbanismo y diseño arquitectónico, así como el surgimiento de expresiones contestatarias de rebeldía juvenil y de vanguardia artística.

\section{Proyecto 6}

\section{Convergencia o divergencia en los procesos de cooperación al desarrollo para la construcción de gobernabilidad multinivel en Centroamérica.}

\section{Unidades Responsables}

- Departamento de Ciencias Jurídicas.

- Departamento de Sociología y Ciencias Políticas.

\section{Equipo de Investigación}

- Roberto Rodríguez Meléndez, Coordinador, Departamento de Ciencias Jurídicas.

- Álvaro Artiga González, Departamento de Sociología y Ciencias Políticas.

- Otilio Miranda, S.J., Departamento de Ciencias Jurídicas.

- Carolina Quinteros, Departamento de Sociología y Ciencias Políticas. 


\section{Resumen}

El presente estudio sobre los procesos de cooperación al desarrollo en Centroamérica como factor para la construcción de la Gobernanza Multinivel, tiene como punto de partida metodológico el análisis de dichos procesos y su efecto a nivel regional, nacional y rural territorial. El estudio está centrado en los proyectos de investigación aplicada, financiados por la Fundación Carolina de España. En el análisis se aborda el tema de seguridad ciudadana en Centroamérica y se intenta relacionar tres elementos: el apoyo de la Cooperación Española a la Estrategia de Seguridad en Centroamérica (ESCA), los retos que ella representa, y los niveles de desarrollo que, desde los ámbitos nacionales, se ha otorgado en materia de seguridad ciudadana. El punto central del trabajo es la identificación de "puertas" o "vasos comunicantes" entre los procesos nacionales y regionales, a efecto de identificar buenas prácticas que permitan consolidar lo que se ha denominado Gobernanza Multinivel con el aporte de la cooperación internacional en Centroamérica.

\section{Proyecto 7}

\section{Monitorización de variables físico-químicas en humedales RAMSAR de El Salvador.}

\section{Unidades responsables}

- Departamento de Electrónica e Informática.

- Departamento de Ingeniería de Procesos y Ciencias Ambientales.

\section{Equipo de Investigación}

- Mauricio Pohl, Departamento de Electrónica e Informática.

- Dolores Rovira, Departamento de Ingeniería de Procesos y Ciencias Ambientales.

- Nelly Amaya, Departamento de Ingeniería de Procesos y Ciencias Ambientales.

- Carlos Juárez, Departamento de Electrónica e Informática.

- Héctor Escobar, Departamento de Electrónica e Informática.

- Lissbeth Rodríguez, Departamento de Electrónica e Informática.

- Jordi Jiménez, Fundación CIMNE Latinoamérica.

- Alberto Tena, Fundación CIMNE Latinoamérica.

- Pedro Arnau, Fundación CIMNE Latinoamérica 
- Javier Piazzese, Fundación CIMNE Latinoamérica.

- Julià Sempere, Institut Quimic de Sarrià.

- Rosa Nomen, Institut Quimic de Sarrià.

- Enrique Barraza, Ministerio de Medio Ambiente y Recursos Naturales.

\section{Resumen}

En el presente trabajo se aborda la metodología y la evaluación de tecnologías para realizar una mejor monitorización de los Humedales Ramsar de El Salvador. Se utilizó como caso de análisis el Embalse Cerrón Grande. Desde el 22 de noviembre de 2005, el Embalse Cerrón Grande es nombrado "Humedal de Importancia Internacional Ramsar". A partir de entonces, las 60,698 hectáreas que lo conforman son consideradas como zonas protegidas. Es por ello que con esta investigación se propone una metodología de medición de variables físico-químicas del humedal, con el objetivo de una mejora en la continuidad y sistematización de la información del humedal. Como resultado se obtuvo la metodología a seguir para caracterizar los humedales. Se han obtenido las primeras mediciones a lo largo de todo el humedal Cerrón Grande en el período seco. Se tienen los mapas a lo largo de todo el humedal de temperatura, conductividad, $\mathrm{pH}$ y Oxígeno Disuelto, para ello se han utilizado herramientas de interpolación y simulaciones de fluido dinámica. Además, se han documentado algunos problemas propios del Embalse Cerrón Grande, como aves migratorias que se han vuelto moradoras del lugar, los desplazamientos de tierras debido a que el embalse es utilizado para acumular agua para luego producir energía eléctrica, asimismo evidenciamos la contaminación proveniente de la zona urbana, entre otras. Finalmente, al cierre del documento se presentan dos tipos de propuestas para mejorar el actual sistema de monitorización de los humedales.

\section{Proyecto 8}

\section{Las marcas territoriales como estrategia de posicionamiento y diferenciación: creación de una marca de calidad territorial común.}

\section{Unidades responsables}

- Departamento de Administración de Empresas.

- Departamento de Sociología y Ciencias Políticas. 


\section{Equipo de Investigación}

- Ernesto Zablah, Departamento de Administración de Empresas.

- Sergio Bran, Departamento de Sociología y Ciencias Políticas.

- Vitelio González, Departamento de Administración de Empresas.

- Fernando Villatoro, Departamento de Sociología y Ciencias Políticas.

\section{Resumen}

Este proyecto se propone aportar conocimiento al proceso de revalorización de los territorios, en relación con la construcción y posicionamiento de marcas territoriales como estrategia de agregación de valor. Con esta estrategia se procura construir ventajas que permitan la diferenciación de los productos en los mercados, facilitando con ello el uso -en términos comerciales- de la identidad, el saber hacer y las particularidades de la zona costera del departamento de La Libertad. De esta manera se pretende identificar potencialidades sociales, culturales, económicas, productos y marcas territoriales en la zona costera del departamento de La Libertad. Se elaboró un mapa de marcas, recursos, saberes y haceres territoriales en El Salvador. También se diseñó un protocolo (modelo) para la construcción y desarrollo de marcas territoriales aplicable a todos los territorios involucrados en el proyecto que permitirá elaborar un instrumento de estrategia mercadológica que promueva la competitividad territorial.

\section{Proyecto 9}

\section{Evaluación de la calidad metalúrgica de aceros herramientas y aceros rápidos tratados térmicamente en atmósfera inerte.}

\section{Unidades responsables}

- Departamento de Mecánica Estructural.

- Departamento de Electrónica e Informática.

- Departamento de Ciencias Energéticas y Fluídicas.

\section{Equipo de Investigación}

- Manuel Amador Pineda Campos, Departamento de Mecánica Estructural.

- Mario Chávez, Departamento de Ciencias Energéticas y Fluídicas.

- Víctor Javier Figueroa, Departamento de Electrónica e Informática. 
- Rodrigo Orlando Gonzales Echeverría, estudiante de Ingeniería Mecánica.

- Josué David Salvador Rugamas, estudiante de Ingeniería Mecánica.

\section{Resumen}

En esta investigación se realizó la evaluación metalúrgica de dos tipos diferentes de aceros herramientas: AISI O1 y AISI D2, que fueron tratados térmicamente en atmósfera inerte y templados en aceite. El tratamiento térmico en atmósfera inerte de las muestras de acero se realizó en un horno de gas propano diseñado y construido en el Laboratorio de Ciencias de los Materiales, dentro de las instalaciones de la UCA, para una temperatura máxima de $1200^{\circ} \mathrm{C}$. Dicho horno ha sido diseñado para trabajar con un quemador atmosférico y aire comprimido.

Las muestras de acero se colocaron en el interior de la recámara de acero inoxidable que es parte del horno de gas propano, y dentro de la cual se inyecta gas nitrógeno a $6 \mathrm{~L} / \mathrm{min}$., para generar una atmósfera inerte. La importancia de los resultados en los tratamientos térmicos de las muestras de acero herramienta está ligada al hecho de que, actualmente, se inician investigaciones junto con el desarrollo de tecnología nacional en hornos y tratamientos térmicos dentro de los laboratorios de la universidad. Una vez haya concluido totalmente el proyecto, el laboratorio podrá brindar asesoría y consultoría en hornos y tratamientos térmicos. Finalmente, este desarrollo tecnológico permitirá que la universidad organice cursos teórico-prácticos para docentes, investigadores, profesionales y alumnos.

\section{Proyecto 10}

\section{Construcción e implementación de planes de ordenamiento y desarrollo territorial en El Salvador: capacidades de los actores en el Área Metropolitana de San Salvador (AMSS) y la región Los Nonualcos 2000-2012.}

\section{Unidades responsables}

- Departamento de Sociología y Ciencias Políticas.

- Departamento de Organización del Espacio. 


\section{Equipo de Investigación}

- Andrew Cummings, Coordinador, Departamento de Sociología y Ciencias Políticas.

- Carlos E. Ferrufino, Co-coordinador, Departamento de Organización del Espacio.

- Sergio Bran, Investigador, Departamento de Sociología y Ciencias Políticas.

- Sofía Escoto, Investigadora, Departamento de Sociología y Ciencias Políticas.

- Patricia Fuentes, Investigadora, Departamento de Organización del Espacio.

- Ricardo Ramos, Investigador, Departamento de Organización del Espacio.

- Tatiana Espinal, Asistente de investigación, Departamento de Organización del Espacio.

- Tania Muñoz, Asistente de investigación, Departamento de Sociología y Ciencias Políticas.

- Julio Nájera, Asistente de investigación, Departamento de Organización del Espacio.

- David Vides, Asistente de investigación, Departamento de Organización del Espacio.

\section{Resumen}

El Salvador ha hecho un importante recorrido en la elaboración e implementación de planes de ordenamiento y desarrollo territorial. En este trabajo interesa reconocer cuáles son las capacidades de planificación aplicadas por los actores territoriales en cuatro experiencias en el Área Metropolitana de San Salvador y en la región denominada como Los Nonualcos. Por otra parte, también interesa identificar los resultados producto del despliegue de esas capacidades y visualizar los factores endógenos y exógenos que condicionan esa aplicación. Para ello se han analizado experiencias a diferentes escalas en Santa Tecla, Apopa, Los Nonualcos y Tecoluca, a partir de una marco conceptual que privilegia una visión sistémica de territorio y una clasificación de las capacidades según su naturaleza política y técnica. Sobresalen los resultados en términos de la construcción de nuevos espacios de gobernanza asociativa, la efectiva vigencia de algunos planes y la inversión pública en proyectos específicos. La efectividad de los procesos parece depender de tres factores específicos: su historia, la continuidad de autoridades públicas y los equipos técnicos, además del liderazgo de cara a la construcción de un proyecto político compartido de desarrollo territorial. 


\section{Proyecto I I}

Sistema constructivo para cubiertas y envolventes livianos de baja conductividad térmica derivados del reciclaje de plásticos.

\section{Unidades responsables}

- Departamento Organización del Espacio.

- Departamento de Operaciones y Sistemas.

- Departamento Mecánica Estructural.

- Departamento Ciencia Energéticas y Fluídicas.

\section{Equipo de Investigación}

- Lizeth Rodríguez, Coordinadora e investigadora, Departamento Organización del Espacio.

- Ana del Pilar Letona, Investigadora, Departamento de Operaciones y Sistemas.

- Florencia Lartategui, Investigadora, Departamento Mecánica Estructural.

- Mario Wilfredo Chávez, Investigador, Departamento Ciencia Energéticas y Fluídicas.

- Lissett Ruiz, Asistente de investigación, arquitecta.

- Jazmin Velásquez, Asistente de investigación, egresada Arquitectura.

- Diana Góchez, Asistente de investigación, estudiante de Arquitectura.

- José Miguel Paz, Asistente de investigación, egresado de Ingeniería Industrial.

- Marlon Medina, Asistente de investigación, egresado de Ingeniería Mecánica.

- Gyzel Pérez, colaboradora, egresada de Ingeniería Mecánica.

- Hugo Salamanca, colaborador, egresado de Ingeniería Industrial.

- Javier Villanueva, colaborador, egresado de Ingeniería Industrial.

- Ronald Pedro Santos, colaborador, estudiante de Ingeniería Mecánica.

- Rina Díaz, colaboradora administrativa, Departamento Organización del Espacio.

\section{Resumen}

En el presente artículo se desarrollan fundamentos tecnológicos que confirman el potencial y la factibilidad para el desarrollo de sistemas y 
materiales constructivos para cubiertas y envolventes, utilizando el plástico reciclado PET como materia prima. Asimismo, se busca generar conocimiento tecnológico por medio de la investigación e implementación de metodologías que contemplan procesos experimentales para la generación de datos bajo condiciones controladas. Esto permitirá la disponibilidad de información que fundamentará la hipótesis de crear nuevos materiales constructivos a partir de material reciclado, además de disminuir la disposición final en los rellenos sanitarios de materiales de biodegradación prolongada. Por otra parte, el proyecto ha representado la posibilidad de transferir conocimiento multigeneracional, multidisciplinario, así como multi institucional. 


\section{Normas de publicación en Realidad}

Realidad publica ensayos, notas y reseñas en idioma español relacionados con las diversas disciplinas de las ciencias sociales y las humanidades. [advertencia sobre los derechos de autor], si se opera bajo licencia de Creative Commons] ver Andamios

La publicación de originales se rige por medio de las siguientes normas:

\section{Presentación de artículos, notas y reseñas}

Los trabajos se remitirán en formato Word como documentos adjuntos de correo electrónico a la siguiente dirección: director.realidad@uca.edu.sv

O a la dirección postal:

Ricardo Roque Baldovinos

Director de Realidad, Revista de Ciencias Sociales y Humanidades

Apartado Postal (01) 168

San Salvador

El Salvador

\section{Presentación de ensayos}

a. El ensayo deberá ser original, inédito y de alta calidad académica.

b. En el caso de los ensayos, estos deberán ir precedidos de dos resúmenes [abstract] de entre 100 y 150 palabras cada uno: el primero, se escribirá en español y el segundo en inglés. También deberán llevar palabras claves en esos dos idiomas. La medida de los márgenes izquierdo, derecho, superior e inferior será de 2,54 cms.

c. El nombre del autor o autores irá consignado después del título del mismo. A continuación, se incluirá el nombre de la universidad o la institución de afiliación.

d. Las palabras en una lengua diferente a la de la redacción del texto aparecerán en cursiva o itálica; asimismo se empleará este tipo de letra para resaltar alguna palabra clave, y cuando esto suceda en un fragmento textual en cursiva, se procederá de modo contrario, i.e., destacar la palabra clave en caracteres normales.

e. Las figuras, ilustraciones y tablas deberán ir numeradas con cifras arábigas y con un pie indicando su contenido (en letra Arial de 10 puntos); se incluirán preferentemente en el documento de Word. De no ser posible, se adjuntarán en archivos separados.

f. La extensión mínima para los ensayos es de 35,000 caracteres y la máxima de 75,000. Para los notas y las reseñas la extensión máxima es 
de 25,000 caracteres. En el conteo de caracteres se incluyen notan y se excluyen espacios en blanco.

\subsection{Tipo y tamaño de letra}

Los originales se presentarán con justificación completa en letra Arial 11 puntos para el texto, la bibliografía, las citas y los resúmenes o abstracts. La letra Arial 10 puntos se requiere para las notas, números sobrescritos, tablas y figuras.

\section{a) Apartados y subapartados}

Los títulos de los apartados se presentarán en negrita, numerados con cifras arábigas, y separados por dos líneas del texto anterior y por una línea del texto siguiente. En cuanto a los títulos de los subapartados, se anotarán en negritas y serán nuevamente numerados (v. gr., 1.1., 1.2., 1.3.), debiendo separarse por una línea tanto del texto que antecede como del texto subsiguiente. Los niveles siguientes, que deberán evitarse en lo posible, serán numerados igualmente con cifras arábigas y se escribirán en texto común (v. gr., 1.1.1., 1.1.2.; 1.1.1.1., 1.1.1.2.).

\section{b) Referencias bibliográficas}

Los autores enviarán sus colaboraciones en los sistemas en referencias bibliográficas APA, MLA y Chicago.

\section{Presentación de comentarios y recensiones bibliográficas}

Tanto los comentarios como las recensiones se regirán por los mismos requisitos en cuanto al tipo y el tamaño de letra. La extensión mínima de los comentarios es de 5,000 caracteres y la máxima, de 15,000. Los comentarios no requieren resumen. Las recensiones de libros irán encabezadas por la ficha técnica del libro (Autor o autores, Título del libro, Ciudad, Año, número de páginas. Número de ISBN). Tendrán un máximo de 12,000 caracteres. Al final de la recensión, se incluirá el nombre del autor o autora de la misma y el nombre de la universidad o institución de procedencia.

\section{Criterios de publicación}

Todos los trabajos sometidos serán arbitrados por pares por los sistemas visto, ciego o doble ciego. No se entablará correspondencia por trabajos no solicitados. 